\title{
Multifuncionalidade da paisagem como subsídio às políticas públicas para o desenvolvimento rural sustentável
}

\author{
Landscape multifuncionality evaluation as a subsidy to public policies for sustainable rural \\ development
}

\author{
Carlos Loch ${ }^{\mathrm{I}}$ Paola Beatriz May Rebollar ${ }^{I^{*}}$ \\ Yuzi Anai Zanardo Rosenfeldt ${ }^{\mathrm{I}}$ Marines Walkoski
}

\section{RESUMO}

O conceito de multifuncionalidade propõe que a agricultura oferece à sociedade funções produtivas, ecológicas e culturais. Políticas públicas que promovam a combinação de diferentes funções podem melhorar o desempenho multifuncional da paisagem rural. O objetivo deste artigo é apresentar um método de avaliação da multifuncionalidade baseado no sistema de valores dos atores sociais locais que possa ser utilizado para embasar a elaboração de políticas públicas de desenvolvimento rural. Este método utilizou um sistema de informações geográficas para identificar os elementos presentes na área de estudo, localizada no município de Joinville, Santa Catarina, entre 2010 e 2012. A partir de entrevistas com 53 pessoas, foram atribuídos valores para 15 descritores que representam as funções produtivas, ecológicas e culturais de cada elemento identificado. Estes valores foram ponderados considerando a porcentagem da área ocupada por cada elemento e seu índice de forma. Os resultados ponderados apresentaram os Cursos d'Água com o melhor desempenho multifuncional em oposição aos Agroecossistemas com o pior desempenho. $O$ método proposto apresenta vantagens, porque permite: a) a quantificação de percepções intuitivas facilitando a interpretação de situações complexas; b) avaliar paisagens rurais em diferentes escalas; c) compreender o desempenho de cada função e o equilíbrio entre as funções da paisagem. Mas, também apresenta fragilidades: a) não é objetivo, uma vez que deriva de pontos de vista; $b$ ) as respostas podem sofrer interferências relacionadas com a atitude do pesquisador; c) os resultados apresentam validade temporal e espacial restrita; d) o comprometimento dos entrevistados define a validade dos resultados.

Palavras-chave: desenvolvimento rural, avaliação da paisagem, políticas públicas.

\section{ABSTRACT}

The concept of multifunctionality suggests that agriculture provides to society productive, ecological, and cultural functions. Public policies that promote a combination of different functions can improve multifunctional landscape performance. The objective of this paper is to present an evaluation method of multifunctional landscapes based on values systems of local stakeholders that can be used to support the development of public policies for rural development. This method used a geographic information system to identify the elements present in the study area located in the city of Joinville, Santa Catarina, between 2010 and 2012. From interviews with 53 people were assigned values for 15 descriptors representing the productive, ecological and cultural features of each element identified. These values were weighted considering the percentage of area occupied by each element and its form index. The weighted results showed the best multifunctional performance with the Water Resources opposed to Agroecosystems. The proposed method has advantages because it allows: a) quantifying the intuitive perceptions facilitating the interpretation of complex situations, $b$ ) assess rural landscapes at different scales, c) understand the performance of each function and the balance between the functions of the landscape. But, it also has weaknesses: a) is not objective, since it derives from viewpoints b) responses may get interference related to the attitude of the researcher $c$ ) the results have limited validity in time and space, d) commitment of respondents define the validity of the results.

Key words: rural development, landscape evaluation, public policies.

\section{INTRODUÇÃO}

A agricultura (cultivos vegetais e produção animal) cobre, aproximadamente, metade da área habitável do planeta (CLAY, 2004). As decisões tomadas pelos agricultores na gestão de seus agroecossistemas são importantes para todos. Historicamente, esta atividade contribuiu além da

'Laboratório de Fotogrametria e Sensoriamento Remoto, Universidade Federal de Santa Catarina (UFSC), 88040-900, Florianópolis, SC, Brasil. E-mail: paola.rebollar@gmail.com.*Autor para correspondência. 
produção de alimentos e fibras, com a preservação de recursos naturais, com a reprodução de complexos culturais e sociais, com a segurança alimentar das famílias produtoras e com a oferta de emprego rural (COSTANZA et al., 1997).

Políticas públicas voltadas para o desenvolvimento rural devem considerar, além do progresso material, o respeito às capacidades e anseios das pessoas, a conservação dos recursos naturais e os benefícios que recebemos dos ecossistemas. Percebese que este desenvolvimento deve ser assimilado como processo de transformação da sociedade em relação às potencialidades e oportunidades, eliminando as limitações e atraindo a comunidade. Para alcançar estes objetivos, são necessárias políticas embasadas em conceitos sólidos, que permitam o planejamento em longo prazo, reconsiderando antigos valores (FURTADO, 1974; SACHS, 2009; VEIGA, 2006).

$\mathrm{O}$ conceito de multifuncionalidade, desenvolvido na França no final do século XX, propõe que as paisagens rurais podem produzir externalidades na forma de commodities (remunerados pelos mercados) e não-commodities (não remunerados) (LAURENT, 1999). As externalidades não remuneradas incluem tanto as funções ecológicas, como sequestro de carbono, biodiversidade e ciclagem de nutrientes, quanto as funções culturais, como entretenimento, preservação histórica e qualidade visual (LOVELL et al., 2010).

As pesquisas realizadas sobre a multifuncionalidade das paisagens rurais brasileiras utilizaram este conceito para a compreensão dos processos existentes no meio rural e apontaram seus desdobramentos para formulação de políticas públicas (CARNEIRO \& MALUF, 2005). No entanto, existem poucas formas de avaliar as múltiplas funções das paisagens rurais. O objetivo deste artigo é apresentar um método de avaliação que permita qualificar e, posteriormente, quantificar a multifuncionalidade a partir do sistema de valores dos atores sociais locais, visando a embasar a elaboração de políticas públicas de desenvolvimento rural.

\section{MATERIAL E MÉTODOS}

Área de estudo

O município de Joinville tem como coordenadas de referência $26^{\circ} 18^{\prime} 05^{\prime}$ 'S e 4850'38'W e é a principal área industrial do Estado de Santa Catarina. Está cercado por áreas protegidas e remanescentes da Floresta Atlântica. A área pesquisada neste artigo está localizada na porção rural deste município.
Foi selecionado um recorte no distrito de Pirabeiraba (7.102.400mS e 7.099.600mS, 707.450mE e $709.500 \mathrm{mE}$ ) com 758,5ha, caracterizado por pequenas propriedades agrícolas familiares representadas por 166 parcelas fundiárias, reconhecidas pela Prefeitura Municipal de Joinville. Esta área representa a transição entre os espaços urbano e rural e está localizada na bacia do rio Cubatão, que abastece com água, 70\% do município, o qual provocou enchentes em tempos passados.

As atividades agrícolas representam 0,5\% da economia do município e estão localizadas nas áreas utilizadas para conservação ambiental (área de proteção ambiental - APA Dona Francisca) e para o turismo rural (roteiros do Quiriri e da Estrada Bonita) (IPPUJ, 2009). Assim, esta área oferece múltiplas funções para a sociedade local e regional.

\section{Avaliação damultifuncionalidade}

O método desta pesquisa consistiu na elaboração de uma ferramenta de avaliação que permitiu qualificar e, posteriormente, quantificar o desempenho multifuncional da paisagem rural da área de estudo. As funções produtiva, ecológica e cultural foram valoradas pelos atores sociais entrevistados a partir de seus pontos de vista fundamentais. A relação entre o perímetro e a área dos diferentes elementos que compõem a paisagem foi utilizada como variável para ponderar os valores numéricos atribuídos para cada função.

A ferramenta de avaliação foi desenvolvida utilizando os seguintes instrumentos e técnicas:

a) Estruturação de Dados Espaciais em um Sistema de Informações Geográficas (SIG): para definir o perímetro e a área dos elementos existentes na paisagem rural da área de estudo. Foram utilizados (i) software Arcgis/Esri, versão 10.0 para processamento dos dados raster e vetoriais; (ii) ortofotos do voo fotogramétrico do ano de 2007, na escala 1:1.000, cedido pela Prefeitura Municipal de Joinville (PMJ); e (iii) base cartográfica vetorizada em meio digital, com referencial geodésico SIRGAS 2000, proveniente de restituição aerofotogramétrica, do ano de 2007 na escala 1:2.000, cedido pela PMJ.

b) Definição dos Descritores para cada Função Agrícola: os descritoresutilizadosnestapesquisaforam adaptados de LOVELL et al. (2010) e validados pelos atores sociais envolvidos. Os descritores avaliados para as funções produtivas foram produtividade/ colheita, eficiência dos insumos, diversificação de produtos, qualidade/especialidade dos produtos e valor econômico da propriedade. Para as funções ecológicas biodiversidade/habitat para animais 
silvestres, baixa aplicação de produtos químicos, sequestro de carbono, qualidade/conservação da água e conservação do solo. Para as funções culturais, moradia, qualidade visual, recreação/entretenimento, preservação histórica e educação/pesquisa.

c) Entrevistas com os Atores Sociais Locais: foram realizadas 53 entrevistas semi-estruturadas com 34 agricultores e 19 técnicos, entre julho de 2011 e março de 2012. As entrevistas foram realizadas individualmente e em grupo, nas propriedades rurais e na sede de instituições locais de gestão territorial (Fundação 25 de Julho, Empresa de Pesquisa e Extensão Agropecuária - Epagri, Secretaria de Planejamento - Seplan). Nas entrevistas, foram obtidos os valores para as funções produtiva, ecológica e cultural.

d) Valoração das Funções Produtiva, Ecológica e Cultural: os valores atribuídos para cada descritor representam a contribuição dos elementos existentes nas propriedades para aumentar ou reduzir cada função. Todos os descritores receberam valores que oscilam entre 2 e -2 , em que 2 representa aumento significativo da função analisada, 1 aumento da função, 0 não apresenta relevância para a função, -1 reduz e -2 reduz significativamente a função analisada. O somatório dos valores atribuídos apontou o desempenho de cada elemento da paisagem e permitiu comparar o equilíbrio entre as diferentes funções analisadas na área de estudo.

e) Ponderação dos Valores para cada Função Agrícola: a ponderação dos valores atribuídos a cada função é necessária porque os elementos que compõem a paisagem apresentam áreas e formas diferentes, tornando distinta sua relevância local. A área de cada elemento foi representada como uma porcentagem do local de estudo. O índice de forma foi calculado através da razão do perímetro real do elemento e do perímetro da circunferência de área equivalente, ( $A=\pi R^{2}$ em que Aé a área do fragmento existente e $\mathrm{R}$ o raio da circunferência de área equivalente; $P=2 \pi R$, em que Pé o perímetro da circunferência de área equivalente ao fragmento analisado). Este índice considera que o círculo é a melhor forma possível para reduzir a influência de cada elemento na paisagem (FROMAN \& GODRON, 1986). Ao calcular o desvio da forma existente em relação a um círculo, pode-se inferir que, quanto mais próximo de 1, menor a influência deste elemento. Na ponderação, o valor atribuído a cada função foi dividido pelo produto da porcentagem da área e do índice de forma. Para os cálculos, foram usados os softwares Arcgis/ Esri 10.0 e Excel/Microsoft 2007. Os resultados desta ponderação foi um gráfico de barras indicando a contribuição efetiva de cada elemento da paisagem para as funções produtiva, ecológica e cultural.

\section{RESULTADOS E DISCUSSÃO}

$\mathrm{Na}$ etapa de Estruturação dos Dados Espaciais da área de estudo em um SIG, foi possível perceber que a paisagem local é composta por 5 elementos principais: Agroecossistemas, Florestas, Cursos d'Água, Estradas e Edificações (Figura 1). Os Agroecossistemas, compostos por pastagens, cultivos anuais e perenes, sistemas agroflorestais e açudes, predominam na área com 305,8ha. As áreas com Floresta apresentam 208,7ha, os Cursos d'Água 16,8ha, as Estradas 19,9ha e a Área Construída 27,4ha. A figura 1 apresenta a área de estudo e os elementos identificados.

$\mathrm{Na}$ etapa de entrevistas com os Atores Sociais Locais, foi possível perceber que as principais esferas de influência local são os produtores rurais, as instituições de gestão territorial públicas e as organizações não-governamentais (ONG). Os produtores rurais são agroecológicos (cujo sistema de produção segue as normas definidas pela Lei Federal 10831/2003) e convencionais (cujo sistema de produção emprega insumos químicos e industrializados), organizados ou não em cooperativas ou grupos de produção (Rede Ecovida, Associação das Agroindústrias Artesanais Rurais de Joinville - Ajaar, Associação dos Proprietários de Terras da Mata Atlântica com Recursos Hídricos - Aproagua, Associação de Turismo Ecorural).

As instituições de gestão territorial públicas existentes e atuantes na área de estudo são a Fundação 25 de Julho (órgão municipal de assistência técnica, extensão e educação rural), a Fundação Turística de Joinville (Promotur), a Fundação de Meio Ambiente de Joinville (Fundema), a Secretaria de Planejamento de Joinville (Seplan) e a Empresa de Pesquisa e Extensão Agropecuária de Santa Catarina (Epagri), em função do seu potencial interventivo e proximidade com a produção agrícola na área de pesquisa. Por fim, as principais $\mathrm{ONG}$ locais são o Centro de Promoção da Agroecologia (Cepagro/ UFSC) e o Comitê de Bacia Hidrografia CubatãoCachoeira.

Os resultados da etapa de Valoração das Funções Produtiva, Ecológica e Cultural apontaram que os maiores valores atribuídos às funções produtivas foram obtidos pelas atividades agrícolas (9); nas funções ecológicas, os maiores valores estavam relacionados aos remanescentes florestais (10); e, nas funções culturais, os maiores valores 


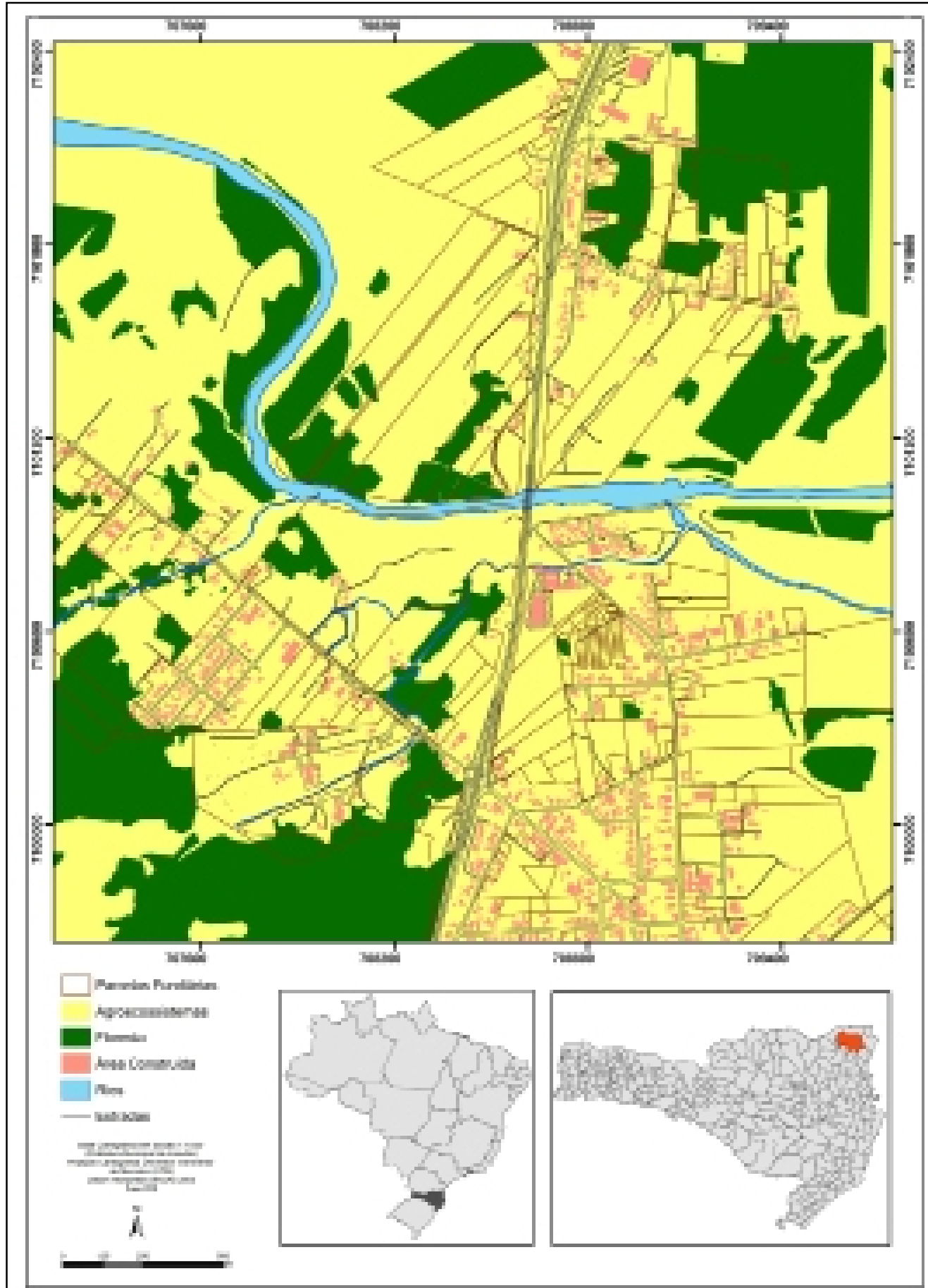

Figura 1 - Os elementos parcelas fundiárias, agroecossistemas, floresta, área construída, cursos d'água e estradas compõem os principais elementos da paisagem da área de estudo.

foram dados às áreas construídas (9). Estes resultados eram esperados, uma vez que o método visa a refletir as percepções intuitivas dos atores sociais envolvidos.

Os pontos de vista dos entrevistados refletidos nos valores atribuídos aos descritores de cada função analisada são influenciados por diversos aspectos. Visando a padronizar estes valores, foi realizada uma ponderação, utilizando o produto da porcentagem da área e o índice de forma de cada elemento existente na paisagem rural. 
Os Agroecossistemas ocupam 40,3\% do local estudado, as Florestas ocupam 27,5\%, os Cursos d'Água 2,2\%, as Estradas 3,6\% e a Área Construída $26,3 \%$. Considera-se que, quanto maior a área ocupada por um elemento, maior sua influência na paisagem. O Índice de Forma calculado foi 1,8 para os Agroecossistemas, 1,6 para a Floresta, 2,4 para os Cursos d'Água, 2,3 para as Estradas e 1,3 para a Área Construída. Neste caso, o índice aponta que, quanto maior o valor absoluto obtido por um elemento, maior sua influência na paisagem.

Depois da ponderação, os Cursos d'Água apresentaram o melhor desempenho nas funções produtivas $(0,56)$, ecológicas $(0,56)$ e culturais $(1,51)$. A Floresta também apresentou valores positivos para todas as funções. Já a Área Construída, Agroecossistemas e Estradas apresentaram bom desempenho nas funções produtivas e culturais, mas receberam valores negativos para as funções ecológicas. A figura 2 apresenta os resultados ponderados da valoração das múltiplas funções.

O desempenho apresentado pelos

Agroecossistemas sinaliza aos gestores públicos a necessidade de incluir o estímulo às funções ecológicas e culturais como objetivos das políticas públicas agrícolas locais. Como apontado por MALUF (2003), “o enfoque da multifuncionalidade valorizaria o fomento à agricultura familiar, porém, através de uma abordagem multifacetada e de instrumentos diferenciados em relação às tradicionais políticas de crédito à produção".

No que se refere especificamente às funções ecológicas, apesar da existência de um conjunto de leis brasileiras visando a melhorar o desempenho ecológico das áreas rurais, a configuração topográfica e fundiária de Santa Catarina e da área de estudo dificulta a aplicação desta legislação. A aplicação estrita das leis implicaria a recuperação florestal de porções significativas das pequenas propriedades, causando o empobrecimento dos agricultores familiares (NEUMANN \& LOCH, 2002). Segundo LOCH (1993), a maioria dos conflitos legais quanto aos problemas ambientais nos minifúndios são causados porque a estrutura fundiária foi projetada em estilo xadrez, incoerente com o relevo acidentado de Santa Catarina. A estrutura fundiária deveria ter sido projetada conforme as microbacias hidrográficas. A utilização do conceito de multifuncionalidade em políticas públicas agrícolas enfatiza uma lógica de planejamento baseada na

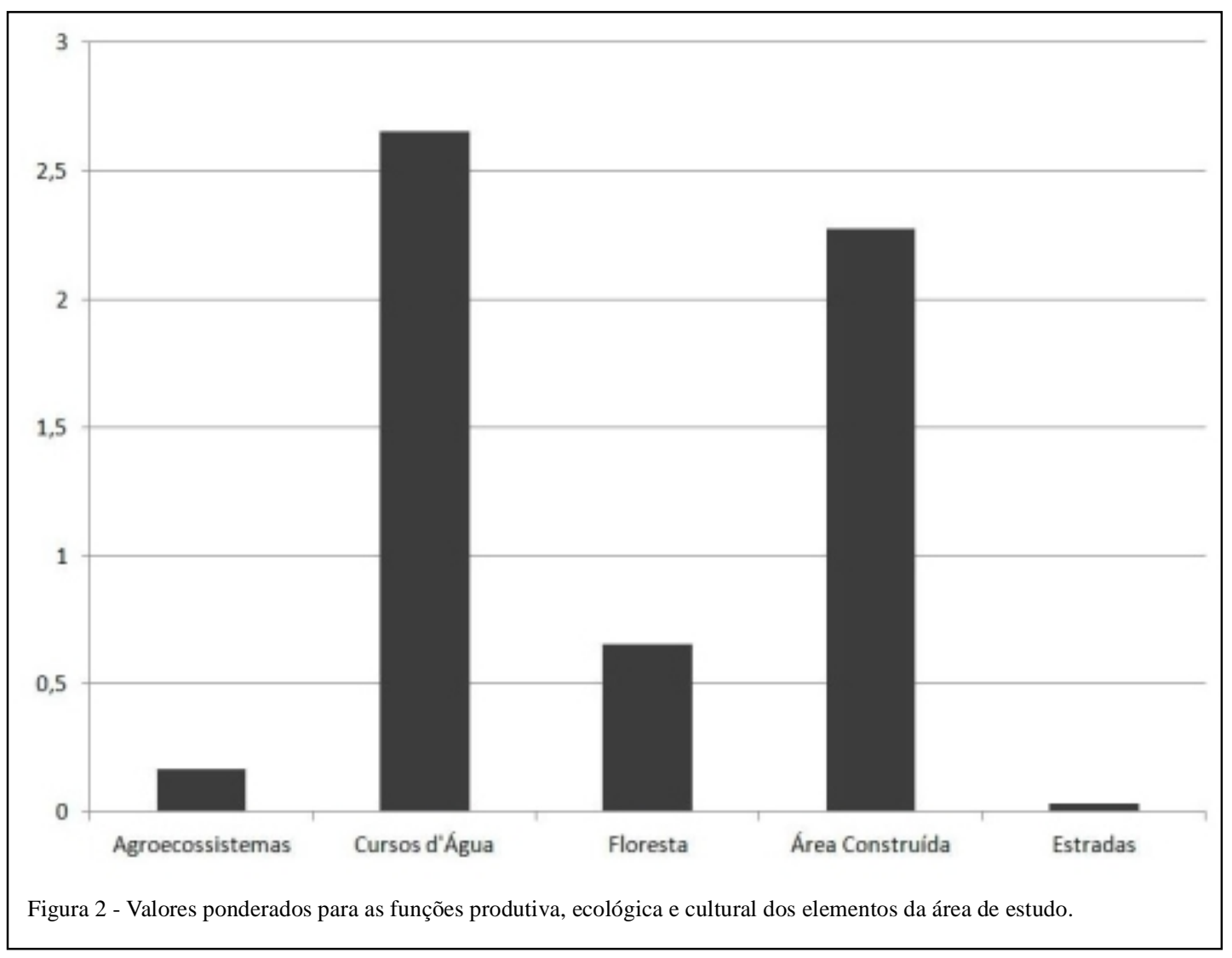

Ciência Rural, v.45, n.1, jan, 2015. 
parcela fundiária, na família e no território. A elaboração de políticas públicas pautadas no conceito de multifuncionalidade estimula os agricultores a se dedicarem com maior compromisso na melhoria do desempenho das funções culturais e ecológicas, recebendo uma remuneração por estas ações (CARNEIRO \& MALUF, 2005). Esta remuneração poderia ser obtida pelo desenvolvimento de atividades como o turismo ou ainda pelo pagamento de serviços ecossistêmicos (MURADIAN et al., 2010; PETHERAM \& CAMPBELL, 2010).

No que se refere ao método proposto, é importante compreender que a gestão pública de espaços rurais é complexa. Em geral, problemas complexos não podem ser analisados a partir de métodos de pesquisa operacional tradicional, que se apóiam em um ou poucos critérios, principalmente medidas quantitativas de eficiência econômica. Da mesma forma, problemas complexos dificilmente podem ser resolvidos através da melhor alternativa técnica, porque envolvem aspectos subjetivos (sistemas de crenças, valores e comportamentos) relacionados com os envolvidos na gestão (ENSSLIN et al., 2001).

O método proposto permite a quantificação de percepções intuitivas dos atores sociais locais. Em muitos casos, mesmo que estas percepções sejam evidentes, a impossibilidade de mensurá-las dificulta sua aplicação na elaboração de políticas públicas. O uso do método proposto permite a geração de conhecimento sobre a área de estudo, que pode embasar os raciocínios de todos os envolvidos na gestão local. Este conhecimento pode auxiliar os atores na compreensão da repercussão que as políticas públicas exercem sobre seus valores (ENSSLIN et al., 2000).

Como todos os métodos de pesquisa, o método proposto apresenta limitações quanto aos resultados que produz. É importante levar em conta que, apesar de propor a quantificação das informações, este método não é puramente objetivo, já que consideram juízos de valores dos envolvidos. Os resultados deste método podem considerar a existência de diversas soluções alternativas para o mesmo problema, de acordo com os sistemas de valores, crenças e comportamentos dos envolvidos (SANNEMANN, 2006).

A objetividade dos resultados obtidos pode ser afetada também pela postura/atitude do pesquisador, que pode influenciar nas respostas e nos posicionamentos dos envolvidos. É importante que o pesquisador assuma uma postura de esclarecimentos sobre o método e os objetivos da pesquisa e sobre o problema em questão. O papel do pesquisador deve ser auxiliar os envolvidos na compreensão da situação ao invés de apontar respostas (ROY, 1993).

Os resultados obtidos a partir do método proposto são limitados no tempo e no espaço. Uma vez que consideram, além de aspectos técnicos objetivos também o conjunto de crenças, valores e comportamentos, os resultados apresentam validade de médio prazo, porque estes aspectos subjetivos mudam ao longo do tempo. Tais aspectos são específicos de cada lugar, logo os resultados obtidos não podem ser extrapolados para outros locais.

Visando a aprimorar os aspectos positivos e minimizar os negativos, é interessante a mobilização dos atores sociais envolvidos. Quando os atores estão conscientes dos objetivos e benefícios que podem derivar da avaliação da multifuncionalidade as respostas, tem maior chance de refletir a situação real da área de estudo em diferentes escalas e existe menor possibilidade de o pesquisador interferir nos resultados.

\section{CONSIDERAÇÕES FINAIS}

A utilização do conceito de multifuncionalidade no planejamento da paisagem rural e na elaboração de políticas públicas implica a superação da perspectiva puramente econômica através da integração de objetivos produtivos, ecológicos e culturais, em que todos são igualmente importantes.

A aplicação do método proposto, baseado na conversão de dados qualitativos em quantitativos, aparece como a inovação deste artigo. Através deste método, é possível apresentar dados confiáveis e de fácil compreensão aos gestores públicos e à sociedade. Estas informações permitem o diagnóstico da situação presente em determinado espaço rural, apontando as potencialidades e fragilidades locais. Além disso, o método pode servir como um ponto de partida para estudos mais profundos, trazendo novas perspectivas aos gestores públicos, que necessitam de ferramentas e parâmetros para subsidiar e mensurar os impactos das ações e políticas adotadas no território.

Considera-se que o método proposto permite avaliar com confiabilidade a multifuncionalidade de uma paisagem rural, quando aplicado corretamente. No entanto, ainda são necessárias novas investigações visando a estabelecer estratégias para operacionalizar a aplicação de uma política de fomento às múltiplas funções da agricultura. É necessário definir, por exemplo, como podem ser feitos os acordos entre agricultores, sociedade e Estado e como podem ser obtidos os recursos para o pagamento das funções ecológicas e culturais desenvolvidas nas propriedades rurais. 


\section{REFERÊNCIAS}

CARNEIRO, M.J.; MALUF, R.S. Multifuncionalidade da agricultura familiar. In: BOTELHO FILHO, F.B. (Org.). Agricultura familiar e desenvolvimento territorial, contribuições ao debate. Brasília: Editora da UnB, 2005. p.43-58.

CLAY, J. World agriculture and the environment. Washington: Island, 2004. 570p. Total de p.570.

COSTANZA, R. et al. The value of the world's ecosystem services and natural capital. Nature, v.387, p.253-260, 1997.

ENSSLIN, L. et al. MCDA: a constructivist approach to the management of human resource at a governamental agency. International Transactions in Operational Research, n.7, p.79$100,2000$.

ENSSLIN, L. et al. Apoio à decisão. Metodologia para estruturação de problemas e avaliação multicritério de alternativas. Florianópolis: Insular, 2001. 130p. .

EPAGRI (EMPRESA DE PESQUISA AGROPECUÁRIA E EXTENSÃO RURAL DE SANTA CATARINA), CEPA(CENTRO DE SOCIOECONOMIA E PLANEJAMENTO AGRÍCOLA). Síntese anual da agricultura de Santa Catarina. Florianópolis, 2010. 184p.

FROMAN, R.T.T.; GODRON, M. Landscape ecology. Cambridge: Cambridge University, 1986. 632p.

FURTADO, C. O mito do desenvolvimento econômico. Rio de Janeiro: Paz e Terra, 1974. 117p.

IPPUJ (FUNDAÇÃO INSTITUTO DE PESQUISA E PLANEJAMENTO PARA O DESENVOLVIMENTO DE JOINVILE). Município de Joinville. Joinville: Prefeitura Municipal, 2009. 164p.

LAURENT, C. Activité agricole, multifonctionnalité et pluriactivité. Paris: Grep, 1999. 227p.
LOCH, C. Cadastro técnico rural multifinalitário como base à organização espacial do uso da terra a nível de propriedades rural. 1993, 128f. Tese (Acesso a Classe de Professor Titular) Universidade Federal de Santa Catarina, Florianópolis, SC.

LOVELL, S.T. et al. Integrating agroecology and landscape multifunctionality in Vermont. Agricultural Systems, v.103, p.327-341, 2010.

MALUF, R.S. A multifuncionalidade da agricultura na realidade rural. In: CARNEIRO, M.J.; MALUF, R.S. (Orgs.). Para além da produção. Rio de Janeiro: Mauad, 2003. 230-247

MURADIAN, R. et al. Reconciling theory and practice: An alternative conceptual framework for understanding payments for environmental services. Ecological Economics, v.69, n.6, p.1202$1208,2010$.

NEUMANN, P.S.; LOCH, C. Legislação ambiental, desenvolvimento rural e práticas agrícolas. Ciência Rural, v.32, n.2, p.243-249, 2002.

PETHERAM, L.; CAMPBELL, B.M. Listening to locals on payments for environmental services. Journal of Environmental Management, v.91, p.1139-1149, 2010.

ROY, B. Decusion science or decision-aid science? European Journal of Operational Research, n.66, p.184-203, 1993.

SACHS, I. Caminhos para o desenvolvimento sustentável. Rio de Janeiro: Garamond Universitária, 2009. 96p.

SANNEMANN, R. et al. Reflections on the structuring of the organizational performance problem using an MCDA-C approach from an integrative systemic-synergetic perspective. In: EUROPEAN CONFERENCE ON OPERATIONAL RESEARCH, 21st, 2006, Iceland. Proceedings EURO XXI, Reykjavik, Iceland p.1040-1042.

VEIGA, J.E. Meio ambiente e desenvolvimento. São Paulo: Senac, 2006. 180p. 